\section{Commentary: Man versus machine: Whose side are you on?}

\author{
Tain-Yen Hsia, MD
}

Go is an ancient Chinese board game that is played today exactly as it was more than 3000 years ago. ${ }^{1}$ It is a game with simple rules, in which 2 players take turns putting black and white stones onto a flat piece of wood on which a grid is printed. To win, a player captures or surrounds more territories than the opponent. Despite its simplicity, Go is far more complex than chess, with possible moves and legal positions estimated to be greater than the number of atoms in the known universe. Go is a game more of intuition than anticipation or calculation. Unlike chess, where a computer with sufficient power can forecast all possible outcomes of a move, Go was believed to be beyond the reach of even the most advanced supercomputers, unsolvable in this lifetime. Among its more than 20 million current human players is Lee Se-dol, a 36-year old South Korean legend who was the fifth youngest individual (at age 12 years) to become a professional Go player and the world's top player during the late 2000s. Widely regarded as humanity's best Go player, Lee famously lost to the Google Deep Mind's AlphaGo, 4 games to 1, a landmark event witnessed by 200 million people and so astounding that the journal Science named it runner-up in its 2016 Breakthrough of the Year selection. ${ }^{2}$ Devastated and saddened by his defeat, Se-dol retired from professional play stating, "Even if I become the number one, there is an entity that cannot be defeated." 3

In an effort to customize the Fontan procedure for optimal flow and hemodynamics, Lok and colleagues ${ }^{4}$ have pitted human surgeons against the machine. The authors demonstrate that an extracardiac Fontan conduit recreated based on surgeon's intuition achieved lower power loss than one produced by computer-aided design (CAD).

From the Pediatric Cardiac Surgery, Yale New Haven Children's Hospital, Yale School of Medicine, New Haven, Conn.

Disclosures: Author has nothing to disclose with regard to commercial support.

Received for publication Jan 22, 2020; accepted for publication Jan 23, 2020; available ahead of print Feb 7, 2020.

Address for reprints: Tain-Yen Hsia, MD, Division of Pediatric Cardiac Surgery, Yale School of Medicine, PO Box 208064, New Haven, CT 06520-8064 (E-mail: tain-yen.hsia@yale.edu).

J Thorac Cardiovasc Surg 2020;160:216-7

$0022-5223 / \$ 36.00$

Copyright (c) 2020 by The American Association for Thoracic Surgery

https://doi.org/10.1016/j.jtcvs.2020.01.065

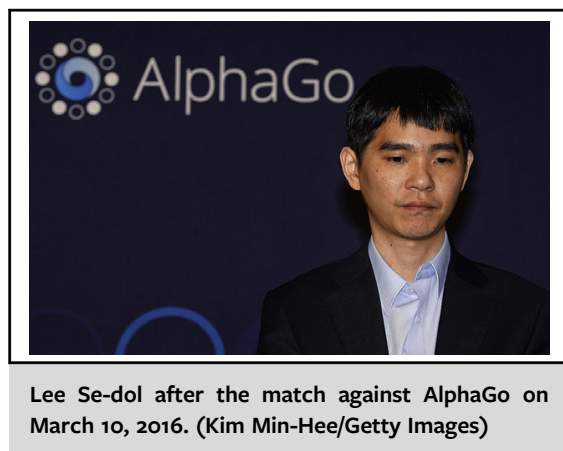

CENTRAL MESSAGE

In the quest for the optimal and

personalized Fontan procedure,

the gap between surgeon intui-

tion and computer-aided design

is closing fast.

However, the CAD Fontan was superior in producing more evenly distributed hepatic venous flow to the lungs and lower shear stresses. The innovative aspects of this study include deconstructing postoperative Fontan geometry/anatomy and allowing off-line redo-Fontan, performing redo-Fontan with a surgeon's free-hand model (using clay) and with $\mathrm{CAD}$, and comparing the flow dynamic performance of the clay and $\mathrm{CAD}$ models using computational fluid dynamics (CFD).

Leveraging CFD to shed light on the importance of Fontan design was introduced by de Leval and colleagues. ${ }^{5}$ It remains the first instance of a change in clinical surgical practice based on computational simulations, by demonstrating the benefit of offsetting the superior and inferior venous connections in a modification of the Fontan. ${ }^{6}$ In those early days, computational methods were simplistic and relied on idealized geometry with nonspecific inputs to yield generalized conclusions. In today's quest for precision medicine and personalized care, the aim is to furnish patient-specific modeling and planning. Therefore, Lok and colleagues ${ }^{4}$ further add to the cumulative insights gained since the first CFD study in 1996. However, whereas in the past we have asked computers to reveal or suggest surgical modifications to improve outcomes, this study is the first to actually ask the question, Can computers perform a better operation than humans? Fortunately (or not, depending on your viewpoint), the study falls short of declaring a winner. After all, its retrospective nature and jousting human versus CAD models in the CFD arena is far from reality and therefore carries little clinical influence. 
How do we know either Fontan construct is possible to implement in a patient? We are fully aware that obligatory adhesions and challenging anatomy (eg, atrial mass, pulmonary vein location, and stuck neoaorta) routinely places constraints on recapitulating ideal connections. As much as we would like to minimize power loss by offsetting an extracardiac conduit from the Glenn, it is almost never possible to get it entirely at surgery. Experiences with the Y-graft Fontan have led to similar complaints, reminding one of the saying, the best-laid plans of mice and man often go awry. We need to know how easy, or difficult, it is to faithfully reproduce an optimal clay or CAD design in the operating theatre. Consequences of deviation by the performed Fontan from the planned should be assessed. A prospective study could examine the feasibility of carrying out a preoperatively designed bespoke Fontan, and evaluate clinical outcomes along with interrogating flow/hemodynamics with postoperative imaging and CFD.

Despite his defeat and the consequent retirement, Sedol remains the only human to ever win a game against AlphaGo in a tournament setting. However, his tournament loss has led to frustration, disappointment, and perceived loss of purpose for many. Immensely complex algorithms such as deep neural networks and Monte Carlo tree search can now endow machines with human qualities such as intuition, creativity, learning, and even selfteaching. When the day comes that a Fontan procedure can be performed by a Da Vinci remote robot upgraded with error-proof artificial intelligence technology, will we surgeons feel the same pang of diminished selfworth as Se-dol? Or will we accept our limitations relative to technology and welcome it as a moment of human growth and triumph? In man versus machine, whose side are you on?

\section{References}

1. American Go Association. A brief history of Go. Available at: https://www.usgo. org/brief-history-go. Accessed February 12, 2020.

2. From AI to protein folding: our breakthrough runners-up. Available at: https:// Www.sciencemag.org/news/2016/12/ai-protein-folding-our-breakthrough-runne rs. Accessed February 12, 2020.

3. Go master retires because AI 'cannot be defeated'. Available at: https://www.bbc com/news/technology-50573071. Accessed February 12, 2020.

4. Lok YH, Kim B, Mass P, Opfermann JD, Hibino N, Krieger A, et al. Role of surgeon intuition and computer-aided design in Fontan optimization: a computational fluid dynamic simulation study. J Thorac Cardiovasc Surg. 2020;160:203-12.e2.

5. de Leval MR, Dubini G, Migliavacca F, Jalali H, Camporini G, Redington A, et al Use of computational fluid dynamics in the design of surgical procedures: application to the study of competitive flows in cavo-pulmonary connections. J Thorac Cardiovasc Surg. 1996;111:502-13.

6. Marsden AL, Feinstein JA. Computational modeling and engineering in pediatric and congenital heart disease. Curr Opin Pediatr. 2015;27:587-96. 